\title{
A Unique Pattern of HCV Genotype Distribution on Hainan Island in China Revealed by Evolutionary Analysis
}

\author{
Tao Wu ${ }^{a, b}$ Lu Xiong ${ }^{c}$ Fuli Wang ${ }^{b}$ Xiaozhen $X^{b}$ Jiao Wang ${ }^{b}$ Feng Lin ${ }^{b}$ \\ Chunhua Lid ${ }^{d}$ Ling Lu ${ }^{d}$ Yuanping Zhou ${ }^{a}$
}

aDepartment of Infectious Diseases, Nanfang Hospital, Southern Medical University, Guangzhou, bDepartment of Infectious Diseases, Hainan General Hospital, Haikou City, cHealth Center, Hainan General Hospital, Haikou City, China; ${ }^{\mathrm{d} C e n t e r}$ for Viral Oncology, Department of Pathology and Laboratory Medicine, University of Kansas Medical Center, Kansas City, KS, USA

\section{Key Words}

Molecular epidemiology $\cdot \mathrm{HCV} \cdot$ Sequence $\cdot$ Evolution $•$ Phylogeographic analysis $•$ China • Hainan Island

\begin{abstract}
Background/Aims: Different genotypes of HCV may differ in both disease progression and response to antiviral therapies. Hainan Island has been inhabited by the "Li" aboriginal minority for centuries. We aimed to provide a better understanding of HCV infection on Hainan Island, so that the information would help improve strategies for HCV prevention and control on the island and in the wider country. Methods: Using RT-PCR and DNA sequencing, we determined HCV sequences from 100 patients living on Hainan Island. Results: Phylogenetic analysis classified these sequences into six subtypes: 6a $(n=35), 1 b \quad(n=31), 3 b \quad(n=16), 2 a$ $(n=8), 3 a(n=6)$, and $1 a(n=4)$. By including reference sequences reported from elsewhere in China, phylogeographic trees were reconstructed to indicate their migration patterns. While the predominant $6 \mathrm{a}$ isolates were estimated to have origins in Guangdong and Guangxi provinces, the increase in $3 \mathrm{~b}$ strains must have resulted from IDU network transmission from the southwest. A Bayesian Skyline Plot for subtype 1a, which is rare in China, showed a rapid population growth since 1998. Although slowed in rate around 2005, this growth continued to the present. Not found for any other HCV lineage. Conclusions: Overall, a delayed growth pattern may indicate the unique history of 1 a dissemination in China and its recently increasing prevalence, despite measures taken to improve HCV prevention.
\end{abstract}

T. Wu and L. Xiong contributed equally to this work.

Ling Lu MD, PhD

and Yuanping Zhou MD, PhD

KARGER
Center for Viral Oncology, Department of Pathology and Laboratory Medicine, University of Kansas Medical Center, MS3046, 3901 Rainbow Boulevard, Kansas City 66160, KS, (USA) and Department of Infectious Disease, Nanfang Hospital Southern Medical University, Guangzhou 510515, Guangdong (China)

Tel. +1 9139456279, +86 13725200272, E-Mail llu@kumc.edu / yuanpingzhou@163.com 


\begin{tabular}{|c|c|c|}
\hline Cellular Physiology & Cell Physiol Biochem 2016; & $39: 316-330$ \\
\hline and Biochemistry & $\begin{array}{l}\text { DOI: } 10.1159 / 000445626 \\
\text { Published online: June 29, } 2016\end{array}$ & $\begin{array}{l}\text { O } 2016 \text { The Author(s). Published by S. Karger AG, Basel } \\
\text { www.karger.com/cpb }\end{array}$ \\
\hline
\end{tabular}

\section{Introduction}

Hepatitis $\mathrm{C}$ virus (HCV) is one of the major causes of chronic hepatitis, liver cirrhosis, and hepatocellular carcinoma [1]. Based on genetic diversity, this virus is now classified into seven genotypes, 97 subtypes (including 84 confirmed and 13 provisionally assigned), and $>20$ lineages that display nucleotide differences equivalent to new subtypes but unassigned $[2,3]$. Different genotypes of HCV may differ in both disease progression and response to antiviral therapies [4-6]. In China, a country with one-fifth of the world's population, HCV is the second major etiology of viral hepatitis, giving rise to viremia in $1.3 \%$ of adults, corresponding to 8.9 (2.7 13.4) million people nationwide. Across all age groups, however, these figures are $0.89 \%$ and 14.8 (4.4 22.3) million, respectively [7]. Currently, there is neither a vaccine nor a post-exposure prophylactic treatment that is effective in preventing $\mathrm{HCV}$ infection, largely because of the high genetic diversity among the viral lineages [8]. Longterm isolated circulation has enabled the virus to accumulate variations to evade population immunity. Modern transmission into naïve people produces new variants that further hamper efforts to control or prevent the infection. There remains a critical need for a better understanding of HCV evolution and migration trends, particularly in geographic regions such as Hainan Island in China where HCV infection represents a major health problem.

Different genotypes of HCV show unique patterns of geographic distribution. Generally, genotypes 1, 2, and 3 are ubiquitous, genotypes 4 and 5 are restricted to Africa [3], and genotype 6 is endemic in Southeast Asia [9]. However, such patterns are evolving as a result of modern transmission and globalization [10]. Recently, a wide-ranging study of HCV infection in China was reported, in which HCV was genotyped in 997 patients from 28 university hospitals located in different geographic regions all in the mainland part of the country. It was revealed that subtype $1 \mathrm{~b}$ is predominant $(56.8 \%, 582 / 997)$, followed by genotype 2 (24.1\%, 240/997), 3 (9.1\%, 91/997), and 6 (6.3\%, 63/97) [11]. However, due to imbalanced sample sizes and different levels of economic development, the frequency of certain HCV lineages was not comparable among the regions studied. Furthermore, there were no samples from Hainan Island, which is separated from the Leizhou Peninsula of mainland China by the Qiongzhou Strait and is located in the South China Sea close to Southeast Asian countries (Fig. 1).

Hainan Island has been inhabited by the "Li" aboriginal minority for centuries [12] and this characteristic, together with its unique geographic location, makes it likely that the HCV genotype distribution among the population on this island is remarkably different from that observed in mainland China. In a series of recent studies, we have investigated the molecular epidemiology and evolutionary patterns of HCV in China, using samples obtained from patients, voluntary blood donors, and injection drug users (IDUs). However, the main focus of these studies was on individuals living in the mainland [13-19]. Although some patients from Hainan Island were included, the number was too small to represent [13]. In the current study, we recruited a larger sample of patients, all of whom live on Hainan Island, for a more detailed investigation. We aimed to provide a better understanding of HCV infection on Hainan Island, so that the information would help improve strategies for HCV prevention and control on the island and in the wider country.

\section{Materials and Methods}

Subjects and samples

Serum samples positive for anti-HCV were collected from 143 patients who visited the General Hospital of Hainan Province. This was completed during routine screening of patients from January 2013 to November 2014. Anti-HCV was assayed using third-generation enzyme immunoassay reagents from Kehua Biotech Co. Ltd (Shanghai, China). All subjects were Han ethnic, of whom 66 (64.7\%) were men and $36(35.3 \%)$ women, with ages ranging from 21 to $65(36.96 \pm 11.24)$. The ethical review committee of the General Hospital of Hainan Province approved this study. Guidelines set by this committee were followed closely and written informed consent was obtained from each patient.

\section{KARGER}


Fig. 1. The location of Hainan Island in China. Separated from the Leizhou Peninsula of the mainland part of China by the Qiongzhou Strait, this island is located in the South China Sea in proximity to Southeast Asian countries. It has been inhabited for centuries by the "Li" aboriginal minority in the central southern mountainous region (green), while the "Han" majority occupy the northern coastal region (blank).

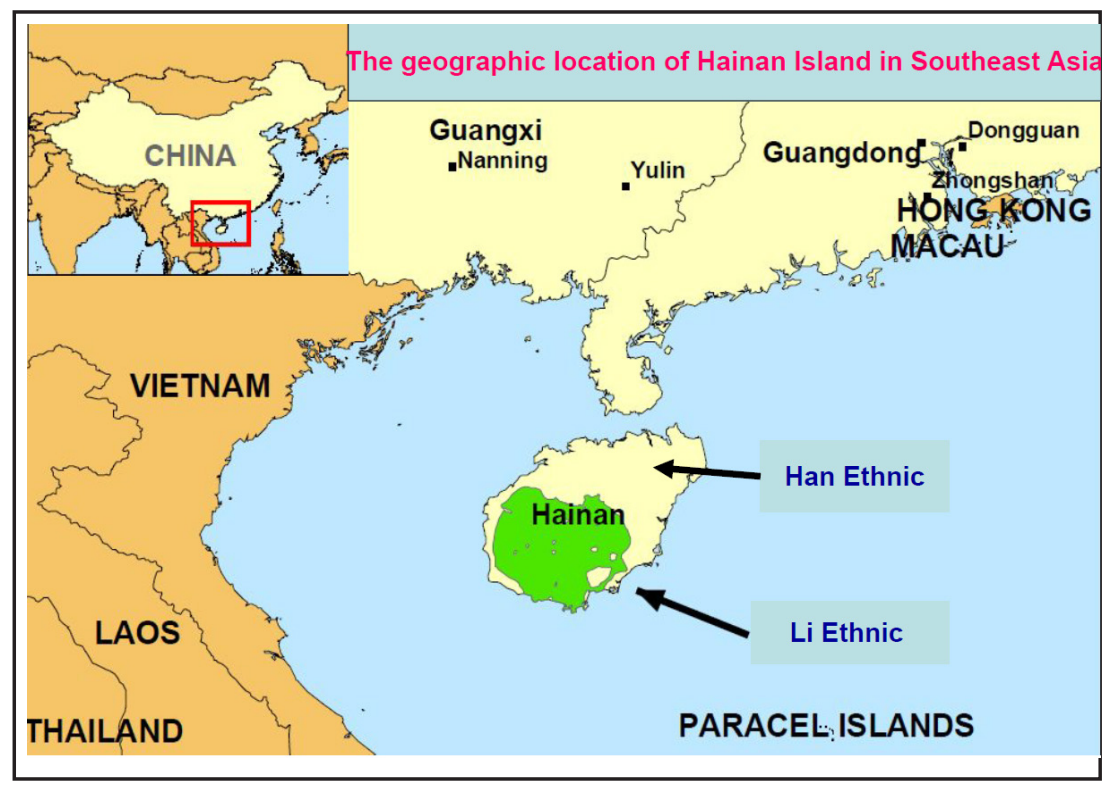

\section{Sequence determination}

HCV sequences from the Core-E1 region (nt 739 to 1310 according to the numbering of the H77 genome) were amplified following previously described protocols [13-18]. Briefly, RNA was extracted in $100 \mu \mathrm{l}$ of serum using the QIA viral RNA reagent (QIAgen); cDNA was converted using random hexamers (Promega) and RevertAid First Strand cDNA Synthesis System (Thermo scientific). Amplification was completed using conventional PCR and the amplicons were purified using the QIAquick PCR Purification Kit (QIAgen). Sequencing was done in both directions using ABI Prism Big Dye 3.0 terminators on an ABI Prism 3500 genetic analyzer (PE Applied Biosystems, Foster City, CA, USA). The resulting chromatograms were visually inspected and sequences were assembled using SeqMan, from which the encoded amino acid sequences were deduced using EditSeq. Sequences were aligned using MegAlign. All software used is contained in the Lasergene 8.1 package (DNASTAR Inc., Madison, WI).

\section{Phylogenetic analysis}

Sequence data sets were assembled based on different HCV subtypes using the BioEdit software (http://www.mbio.ncsu.edu/bioedit/), with the inclusion of reference sequences selected from GenBank to ensure appropriate representation. Prior to phylogenetic tree reconstruction, the best-fitting substitution model was selected using the jModeltest program on the basis of the Akaike information criterion [20]. GTR $+\mathrm{I}+\Gamma$ was found to be the most appropriate model for all resulting sequence data sets. Under this model, the maximum likelihood (ML) trees were heuristically searched using the subtree pruning and regrafting (SPR) algorithm and the nearest-neighbor interchange (NNI) perturbation algorithm implemented in the MEGA6 software [21], with which bootstrap analyses were performed in 1000 replicates. After tree files were generated in the Newick format, tree topologies were displayed using the Fig Tree program [22].

\section{Bayesian coalescence analysis}

We used the Bayesian Markov Chain Monte Carlo (MCMC) algorithm, implemented in the BEAST package [22], to estimate the ancestral relationship and migration patterns of different HCV subtypes detected on Hainan Island. Recent analysis of HCV sequences in the Core-E1 region indicates that the exponential clock model is preferable to the lognormal and strict models [23-25]. Therefore, to analyze the various HCV subtypes, we used the exponential clock model in combination with the GTR $+\mathrm{I}+\Gamma$ substitution model and the Bayesian skyline model. However, prior evolutionary rates differed among HCV subtypes, as we have recently described $[13,17]$. MCMC chains were run for 300 million states and a tree was generated for every 10,000 states. To assess the sampling convergence of the MCMC procedures, the estimated effective sampling sizes (ESSs) were inspected after a 10\% burn-in using the Tracer program (ver.1.5) (http://tree.

\section{KARGER}




\section{Cellular Physiology Cell Physiol Biochem 2016;39:316-330 \begin{tabular}{lll} 
DOI: 10.1159/000445626 & and Biochemistry & $\begin{array}{l}\text { O 2016 The Author(s). Published by S. Karger AG, Basel } \\
\text { wwww.karger.com/cpb }\end{array}$ \\
\cline { 2 - 3 }
\end{tabular} \\ Wu et al.: HCV Distribution Pattern on Hainan Island}

bio.ed.ac.uk). In this study, when all ESSs were $\geq 200$, sufficient sampling was considered to have been achieved. To generate phylogeographic trees in a decreasing node order, all branches were arranged such that the shorter the branches were, the higher they were positioned in the tree and vice versa. The resulting posterior tree files were deciphered using the FigTree program.

Nucleotide sequence accession numbers

The nucleotide sequences reported in this study were deposited in GenBank with the following accession numbers: KX345144-KX345248.

\section{Results}

\section{HCV genotyping}

We successfully determined partial Core-E1 sequences of HCV from 100 patients. With reference to those retrieved from GenBank, we classified these sequences into six subtypes: $1 \mathrm{a}, 1 \mathrm{~b}, 2 \mathrm{a}, 3 \mathrm{a}, 3 \mathrm{~b}$ and $6 \mathrm{a}$. Among them, those of subtype $6 \mathrm{a}$ were predominant $(\mathrm{n}=35)$, followed by $1 b(n=31), 3 b(n=16), 2 a(n=8), 3 a(n=6)$, and $1 a(n=4)$.

\section{Phylogenetic analysis}

Based on these 100 sequences and nine references representing the seven known genotypes of HCV [2], a circular phylogenetic tree was reconstructed (Fig. 2). The 100 new sequences showed great genetic diversity: those of the same subtype were closely grouped, and each group was separated from the others with a full (100\%) bootstrap support.

\section{Phylogeographic analysis}

To indicate genetic diversity and migration trends, BEAST (Bayesian Evolutionary Analysis by Sampling Trees) analysis was performed after including 966 reference sequences. Six time-scaled trees were generated representing subtypes $1 \mathrm{a}, 1 \mathrm{~b}, 2 \mathrm{a}, 3 \mathrm{a}, 3 \mathrm{~b}$, and $6 \mathrm{a}$. These are phylogeographic trees, since information about the geographic regions where these sequences were sampled was also included. Comparison with the tree shown in Fig. 2

Fig. 2. Acircular form of phylogenetic tree.The tree was reconstructed based on the Core-E1 sequences of HCV determined from 100subjects (black) and co-analyzed with standard reference sequences (red) representing subtypes $1 \mathrm{a}, 1 \mathrm{~b}, 2 \mathrm{a}, 3 \mathrm{a}$, $3 \mathrm{~b}, 4 \mathrm{a}, 5 \mathrm{a}, 6 \mathrm{a}$, and $7 \mathrm{a}$. All references shown in red have the format: subtype_isolate. Otherwise, the isolate ID was used as a label. Full (100\%) bootstrap supportis indicated at the most peripheral internal node of each cluster. The horizontal ruler of length 0.2 units per nucleotide per site at the bottom center of the tree acts as a guide to genetic distances.

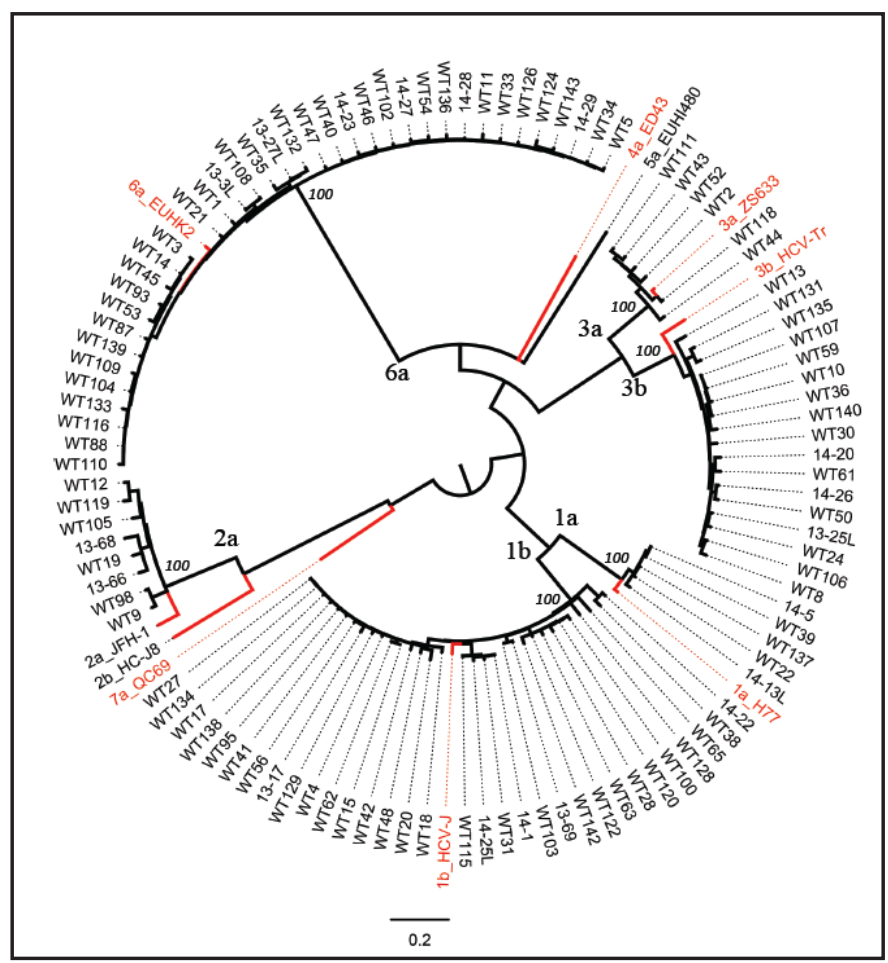




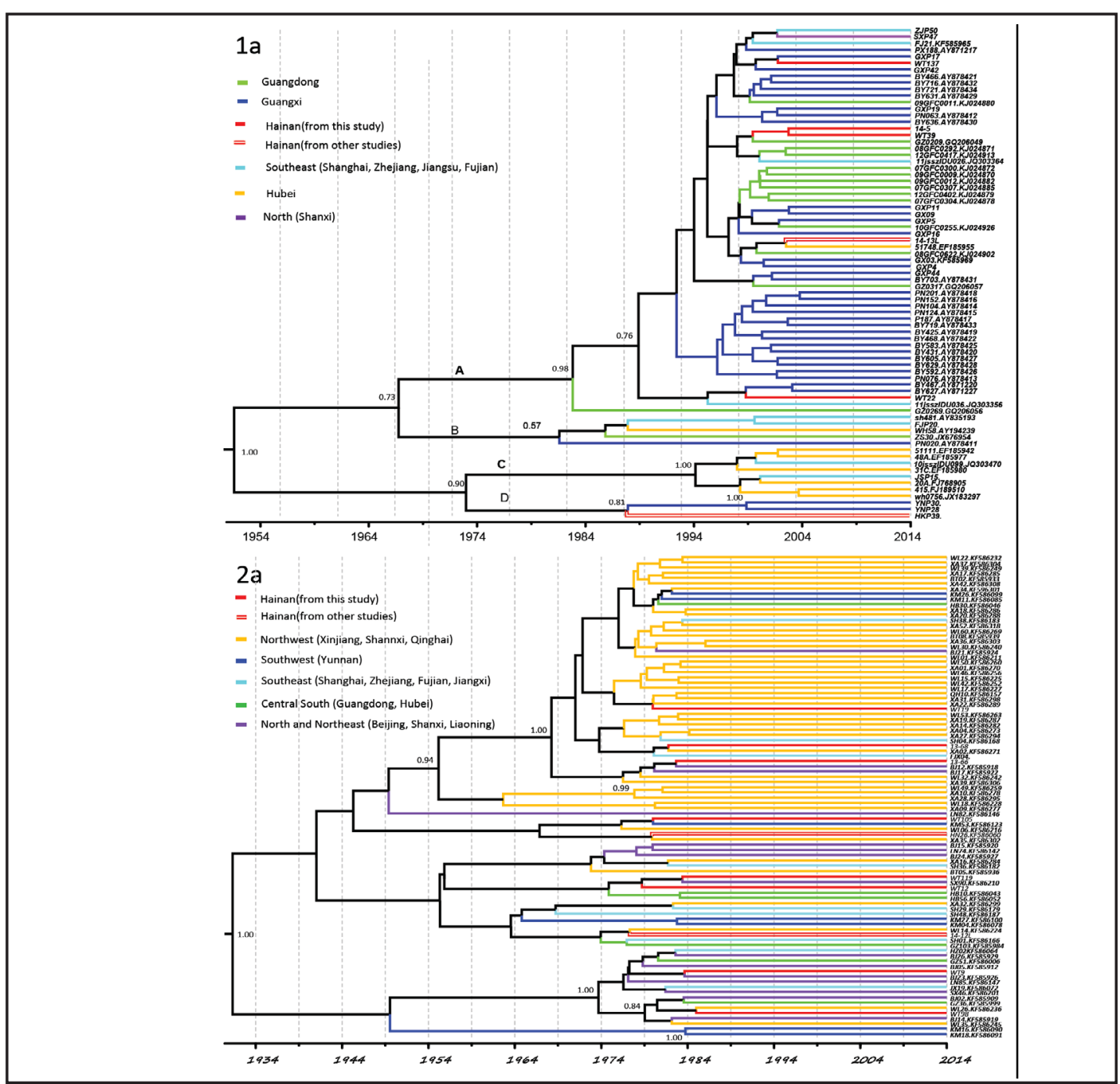

Fig. 3. Phylogeographic trees estimated usingthe Core-E1 sequences of subtypes1a (upper) and 2a (lower). Branches are colored according to their geographic origins, indicated in the upper left. Posterior probabilities of $>70 \%$ are shownat the respective nodes. Below each tree isa time scale from 1954 to 2014 (1a) and from 1934 to 2014 (2a) to indicatethe time of origin and evolution of the respective HCV subtypes.

highlights a remarkable feature of these phylogeographic trees: all their tips were aligned to the right end and linked to branches of considerable length being scaled and colored for a better differentiation. Although not presented, each branch has an associated Bayesian score that measures the likelihood of its divergence.

\section{Subtype $1 a$}

The upper part of Fig. 3 presents a phylogeographic tree based on 75 subtype 1a sequences, comprising four from this study and 71 references from GenBank or from our previous reports. Because the phylogenetic and migration patterns of subtype 1a in China have not been well studied before, we performed a detailed analysis. In this tree, groups A, B, C, and D were tentatively defined with posterior probabilities of $0.98,0.57,1.00$, and 0.81 , respectively. Group A is the major one, containing 59 terminal branches. Because most of the branches had peripheral nodes scaled between 1999 and 2004, a time frame is suggested during which these strains were introduced into China. Interestingly, a large proportion of the references in group A were previously reported in Guangxi $(n=34)$ and Guangdong $(\mathrm{n}=14)$ provinces, which are geographically closer to Hainan Island than other regions. 


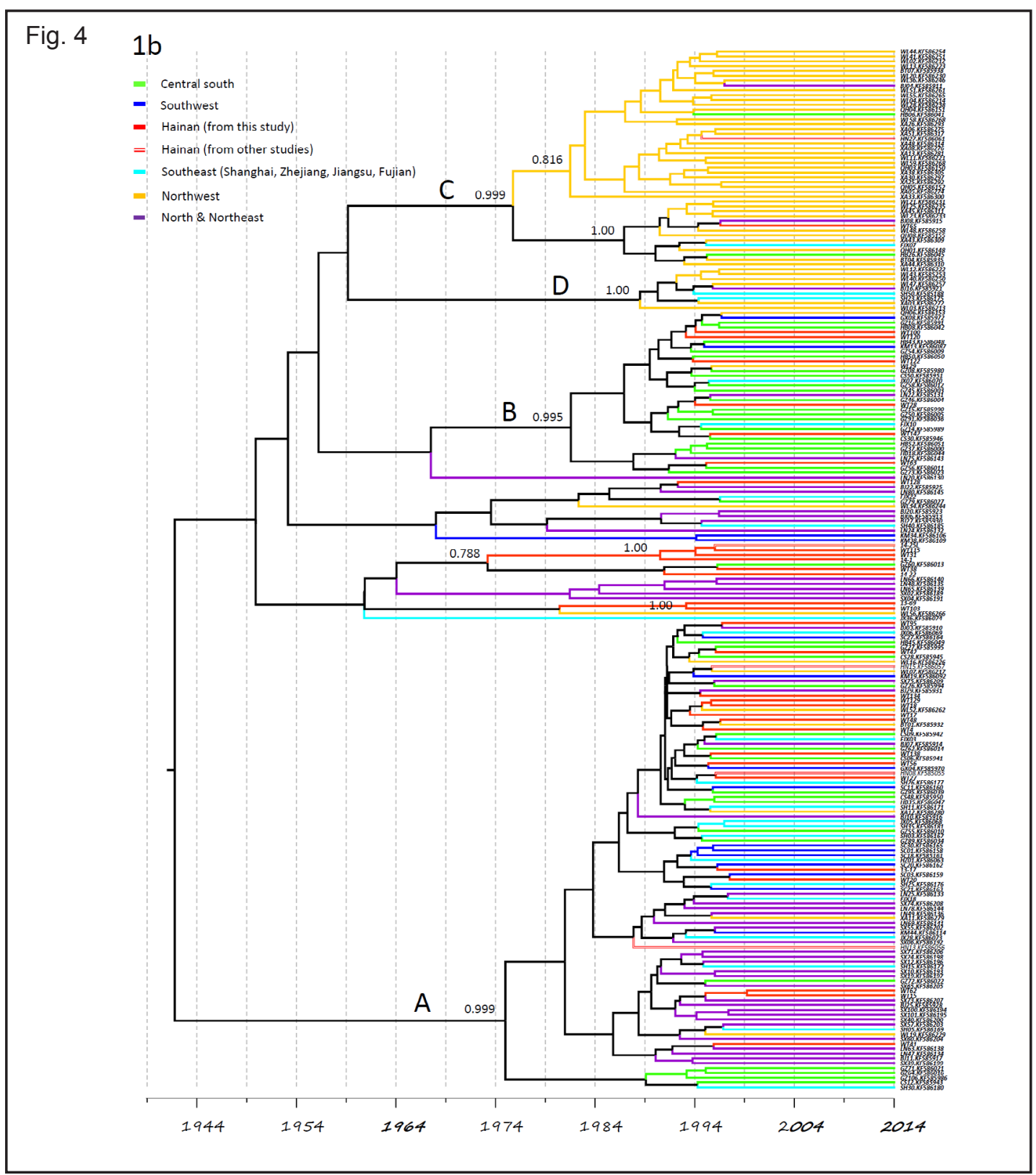

Fig. 4. Phylogeographic trees estimated from the Core-E1 sequences of subtypes $1 b, 3 a, 3 b$, and $6 a$, respectively. Other details as described in Figure 3.

This may explain why the four sequences of $1 \mathrm{a}$ from the present study are all clustered into group A: they may originate from Guangdong or Guangxi provinces. In contrast, only a few reference sequences were reported in other regions. These include two from Jiangsu, and one each from Zhejiang, Fujian, Shanxi, and Hubei $[13,26,27]$. Reference 14-14L was also determined on Hainan Island and segregated into group A, but it was from a patient of the "Li" ethnic minority.

The formation of group B is not robust, possessing a weak posterior probability of 0.57 . Group B includes only five sequences, each from a different province located in the southern half of China: Shanghai, Fujian, Hubei, Guangdong, and Guangxi [18, 28, 29]. These mixed geographic origins may indicate a sporadic pattern.

A characteristic of Group C is that all sequences but one were sampled from IDUs. Of these, six were from Hubei province in the central part of China while two were from Jiangsu 


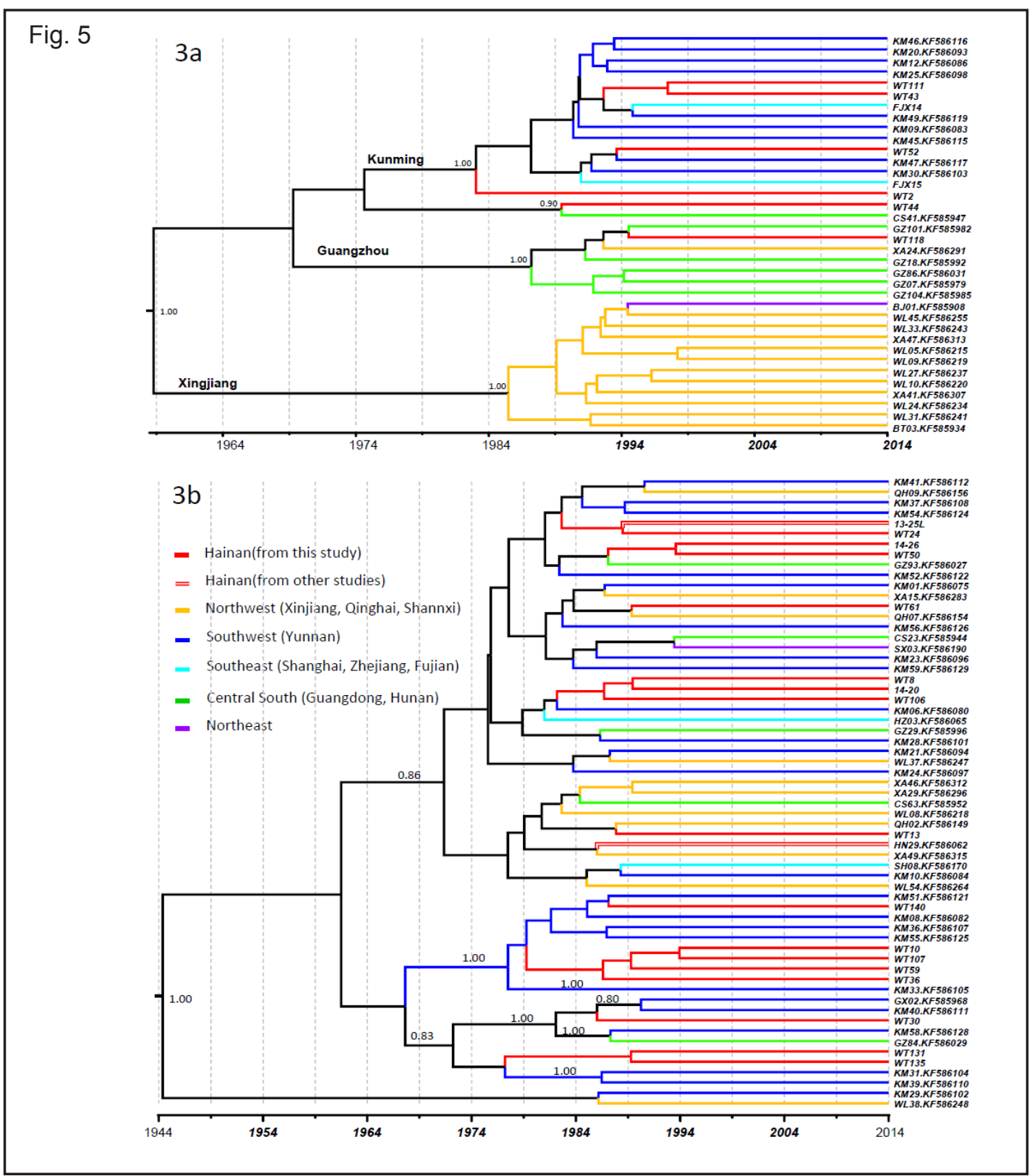

Fig. 5. Phylogeographic trees estimated from the Core-E1 sequences of subtypes $1 \mathrm{~b}, 3 \mathrm{a}, 3 \mathrm{~b}$, and $6 \mathrm{a}$, respectively. Other details as described in Figure 3.

in the southeast $[26,27]$. With its divergence time scaled at around 1994, a long internal branch pairs group $\mathrm{C}$ with group $\mathrm{D}$, which only includes three references: two from Yunnan in the southwest and one from Hainan (from one of our unreported studies). While group $\mathrm{C}$ is thought to be associated with IDUs, no obvious pattern is suggested by group D.

Overall, 75 subtype 1 a sequences were characterized in China to date and they indicate $1 \mathrm{a}$ being mainly prevalent in the south and only one isolate from the north.

\section{Subtype $1 b$}

Thirty-one $1 \mathrm{~b}$ sequences were characterized in this study and analyzed with 216 references, generating the phylogeographic tree shown in Fig. 4. Similar to the tree we previously reported [13], the sequences were divided into four major groups: A, B, C, and D, 


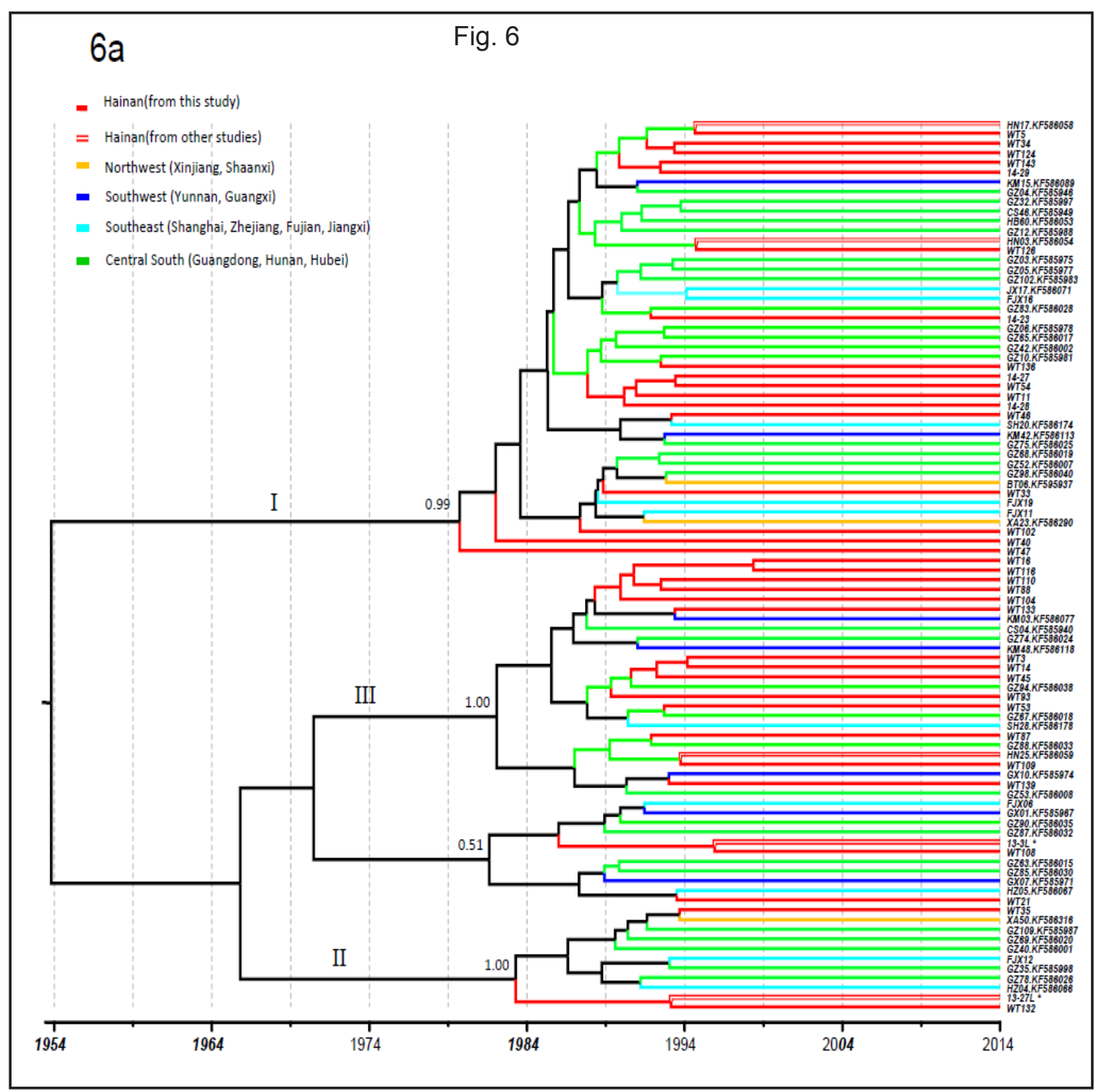

Fig. 6. Phylogeographic trees estimated from the Core-E1 sequences of subtypes $1 \mathrm{~b}, 3 \mathrm{a}, 3 \mathrm{~b}$, and $6 \mathrm{a}$, respectively. Other details as described in Figure 3.

with posterior probabilities of $0.999,0.995,0.999$, and 1.00 , respectively. Of the 31 sequences obtained in this study, 16 were clustered in group A, six in group B, one in group C, but none in group D. As previously described, strains in group A are prevalent nationwide, while those in group B and C are locally epidemic in the central south and northwest of China, respectively. That the majority of the 31 subtype $1 \mathrm{~b}$ sequences from this study segregated into groups $A$ and $B$ is consistent with these patterns. Eight $1 \mathrm{~b}$ sequences segregated outside the A, B, C, and D groups. Of these, five formed a cluster with two references, with a posterior probability of 0.788 , while two formed a twin subset possessing a full posterior probability of 1.00. We recently characterized the GZ60 isolate from a blood donor in Guangdong province [13]. The 14-25L isolate was sampled on Hainan Island, but was from a patient of the "Li" minority (our unpublished data).

\section{Subtype $2 a$}

We analyzed the new subtype 2a sequences with 84 references (see the lower part of Fig. 3). Recently, we constructed a phylogeographic tree based on these 84 references, which shows two major groups: most of the sequences from the larger group were sampled 


\section{Cellular Physiology Cell Physiol Biochem 2016;39:316-330 \begin{tabular}{l|l|l} 
DOI: 10.1159/000445626 & $\begin{array}{l}\text { O 2016 The Author(s). Published by S. Karger AG, Basel } \\
\text { www.karger.com/cpb }\end{array}$
\end{tabular}

Fig. 7. Bayesian Skyline Plot estimated for subtype 1a. The black curve represents the effective population size through time. The upper and lower grey curves define the $95 \%$ highest posterior density confidence interval. A vertical ruler on the left $(Y$ axis) measures the effective population size, while a horizontal scale ( $\mathrm{X}$ axis) at the bottom measures time from 1980 to 2010.

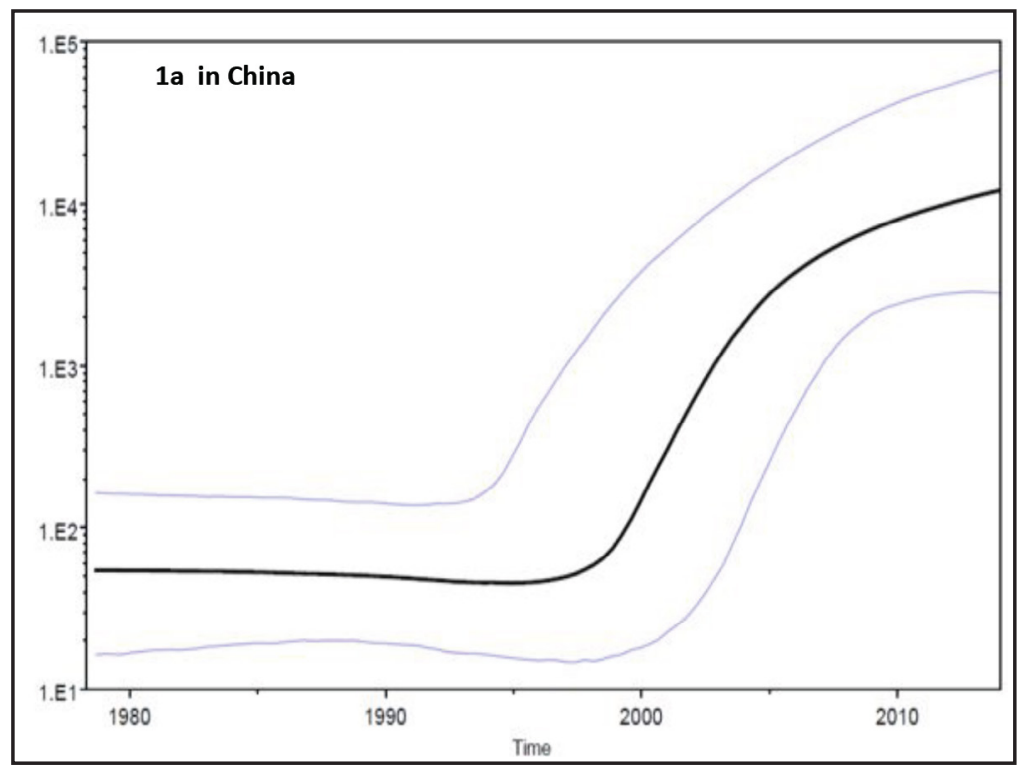

in the northwest, while the smaller group exhibited a mixture of origins. There were also several loose clusters between the two main groups. Of the eight new subtype 2a sequences, three fell into the larger and two into the smaller group, with each showing a full posterior probability of 1.00 . The three remaining sequences fell into the loose clusters.

\section{Subtype $3 a$}

The new subtype 3 a sequences were analyzed with 30 references. The resulting tree (the upper part of Fig. 5) was similar to that we previously reported [13] and showed a very clean structure, containing three separate geographic groups in addition to a twin subset. Each group included references derived almost entirely from a single geographic region and had a full posterior probability of 1.00. We therefore designated them the Kunming, Guangzhou, and Xinjiang groups. Of the six subtype 3a sequences we obtained, four segregated into the Kunming group and one into the Guangzhou group while no sequences partitioned into the Xinjiang group. The remaining sequence, WT44, was paired with reference CS41 to form a twin subset; CS41 was from a blood donor in Hunan province in the central south [13]. This tree appears to suggest that the 3a strains on Hainan Island might have origins in the southwest, likely via IDU network transmission from the Golden Triangle [30].

Subtype $3 b$

Notably, subtype $3 \mathrm{~b}$ represents the third most frequent category of isolates from Hainan Island, having been detected in 16 patients. These sequences were analyzed together with 45 references, allowing us to construct the phylogeny shown in the lower part of Fig. 5 . In contrast to subtype $3 \mathrm{a}$, the tree for $3 \mathrm{~b}$ appeared to be more complex in geographic origin. From the root of the tree, two internal branches diverged. One branch was located at the bottom leading to two references, while the other developed into a clade. Within the clade, two clusters arise, with the upper cluster appearing to be more robust (probability = 0.86 ) than the lower one (probability $=0.30$ ). The references in the lower cluster are more homogeneous in origin than those in the upper one. Of the 16 new subtype $3 \mathrm{~b}$ sequences, eight segregated into the upper and eight into the lower cluster. The time scale suggests that, around 1990, multiple 3b strains were introduced into Hainan Island, possibly originating in southwest China. Subsequently, considerable geographic mixing has occurred.

Subtype $6 a$

In this study, subtype 6a isolates represent the most predominant lineage, detected from 35 patients. Their analysis with 67 references allowed a phylogeographic tree to be 
reconstructed (Fig. 6). Structurally, the tree appeared to resemble that for 3a, but it included references mostly from the central south. Four clusters were identified. Clusters I, II, and III are the same as those we have reported previously $[13,15,17,30]$, each having a full posterior probability of 1.00. In contrast, the fourth cluster is not as robust as these three; it has a probability of only 0.51 and is thus unnamed. Of the 35 new subtype 6 a sequences, 17 were grouped into cluster I, two into cluster II, 14 into cluster III, and two into the unnamed cluster. This pattern supports the hypothesis that the origin of the majority of subtype 6a strains in China was in Guangdong province, although the ancestral origin is likely to be in Vietnam [15]. Within clusters I and III, the sequences from the present study tend to form closely related subsets, suggesting common sources of infection.

\section{Bayesian Skyline Plot (BSP) analysis}

We performed BEAST analysis using 75 subtype 1a sequences shown in Fig. 3. The resulting flexible nonparametric estimate of the past changes in infected population size was illustrated as a BSP (Fig. 7), which showed a relatively constant population size from 1976 to 1998 , followed by a period of rapid growth. Although there was a clear slowing down of the growth rate around 2005, the growth continued through to the present time. After comparison with the BSPs we recently described for subtypes $1 \mathrm{~b}, 2 \mathrm{a}, 3 \mathrm{a}, 3 \mathrm{~b}$, and $6 \mathrm{a}$ [17], two important features of the BSP for 1a become evident: (1) its rapid growth occurred later than that of the other HCV subtypes; and (2) there has been no constant phase in the population size in the near recent past.

\section{Discussion}

This may represent the first detailed report on HCV molecular epidemiology and genotype distribution pattern on Hainan Island. Located in the Tonkin Bay between East Asia and Southeast Asia, this island is characterized by aboriginal inhabitants of the "Li" ethnic minority, among whom multiple new lineages of HCV genotype 6 variants were discovered in one of our recent studies that confirmed the circulation of HCV among the indigenous population on the island over centuries [12]. In the present study, we determined Core-E1 sequences of $100 \mathrm{HCV}$ isolates from Hainan Island and classified them into six subtypes: $1 \mathrm{a}$, $1 \mathrm{~b}, 2 \mathrm{a}, 3 \mathrm{a}, 3 \mathrm{~b}$, and $6 \mathrm{a}$. Although the distribution pattern is largely congruent with what we have defined for HCV in China, differences were observed [13-19]. First, subtype 6a isolates account for the major proportion (35\%). Second, subtype $3 \mathrm{~b}$ isolates occur more frequently $(16 \%)$ than in many other regions of China. Third, subtype 1a isolates were detected in a slightly increased number (4\%) of patients. Fourth, subtype $1 \mathrm{~b}(30.9 \%)$ and $2 \mathrm{a}(9.9 \%)$ isolates were recovered at relatively low frequencies.

In our recent studies using samples collected in Guangdong province, which is close to Hainan Island across the Qiongzhou Strait, subtype $1 \mathrm{~b}$ isolates were found to be predominant. However, the frequency of 6 a isolates has increased in the last decade, and has become the major strain among IDUs and the second most common lineage among patients and voluntary blood donors [13-18]. To date, there has been no large-scale survey of HCV among the general population in Guangxi province, which is bordered by Guangdong to the east and by Vietnam to the south, with Hainan Island across the Tonkin Bay. Among IDUs sampled in Guangxi, 6a isolates are increasingly detected; these are thought to derive from Vietnam, the region of origin of this unique HCV subtype $[15,28]$. Why subtype 6 a has become predominant on Hainan Island remains unknown, but there are four plausible scenarios. First, it may be attributed to the proximity of Hainan to Guangdong and Guangxi provinces and to Vietnam. These three regions may constitute a contiguous geographic area where 6a strains are indigenous. Second, a fraction of the population on Hainan Island are fishermen or descendants of fishermen who are known to travel the coastal regions of Guangdong and Guangxi provinces and the countries of Southeast Asia as well, and thus may be responsible for the introduction of 6a strains. Third, a proportion of the inhabitants of Hainan Island were originally immigrants from other regions of China. Many moved in the 1980s when the island 
was separated from Guangdong as a new province to allow the implementation of special opendoor policies (http://www.npc.gov.cn/wxzl/gongbao/2000-12/05/content_5004499.htm). Among these immigrants, a large number came from the nearby Guangdong and Guangxi provinces where $6 \mathrm{a}$ is prevalent. Fourth, it is possible that subtype $6 \mathrm{a}$ is preferentially linked to IDU transmission; this route may have thus facilitated 6a introduction from neighboring Guangdong province, from where subtype $6 a$ is now spreading nationwide $[13,15]$. Among the four possibilities, the last is supported by the mean age of those patients from whom we isolated 6a sequences: this group was the youngest among our sample (Table 1). However, no clear history of IDU was established for these patients. One may also speculate that the 6 a isolates detected in this study may have origins in niches on Hainan Island, within which indigenous circulation of subtype 6a may have been maintained. Should such niches exist, the 6a sequences obtained in this study ought to display substantial genetic differences from those obtained in other regions. However, extensive phylogenetic analysis did not indicate such differences and instead showed that the new 6a sequences are well integrated with references from other regions of China; no unique variants are detected. The resulting tree is highly similar to that we recently reported, which was based on sequences sampled in 17 provinces/municipalities in China [13]. Such a pattern does not support the hypothesis that 6a strains may have an origin on Hainan Island. However, a definite exclusion of such a possibility would require an extensive survey of HCV to be performed among all ethnic aborigines on Hainan Island. In one of our other studies, we located the ancestral origin of 6a in Vietnam, based on the finding that all 6a sequences from Vietnam are genetically diverse, while those from China and from other parts of the world are relatively homogeneous [15].

In this study, $3 \mathrm{~b}$ isolates were detected in $16 \%$ of cases, making this subtype the second most predominant in our sample. Although not as high as that we reported for subtype $3 \mathrm{~b}$ among patients in Yunnan province in the southwest of China bordering Vietnam and Myanmar [19], the frequency is higher than we expected, which suggests uncharacterized transmission events. In one of our earlier studies completed in 2002, we determined HCV sequences from 139 patients in nine cities across China. In patients from Kunming city, which is the capital city of Yunnan province, $3 \mathrm{~b}$ isolates were isolated in five out of 15 cases (33.3\%). In contrast, no $3 \mathrm{~b}$ isolates were detected in 124 patients from the other eight cities [18]. Recently, we described the genotyping of $411 \mathrm{HCV}$ isolates from blood donors in 17 provinces/municipalities [13]. Among 53 donors from Yunnan, $3 \mathrm{~b}$ isolates were detected in 23 (41.8\%), while among 356 donors from the other 16 provinces/municipalities, 3b isolates were detected in only 11 (3.08\%). In one of our other studies using samples from individuals in Yunnan province, we detected a high frequency $(24 / 80,30 \%)$ of $3 \mathrm{~b}$ isolates among IDUs [19]. Taken together, these three studies suggest that $3 \mathrm{~b}$ strains in China might have origins in Yunnan province. This is also congruent with the results from our other studies, in which $3 \mathrm{~b}$ isolates were detected only in a small proportion of clinical patients $(13 / 393,3.3 \%)$ and voluntary blood donors $(17 / 236,7.2 \%)$ in Guangdong province [14, 16], although Guangdong is close to Hainan. Among countries in Southeast Asia, Vietnam is nearest to Hainan Island across the Tonkin Bay. In Vietnam, the pattern of HCV genotype

Table 1. The demographic information of the sampled patients

\begin{tabular}{cccccccc}
\hline \multirow{2}{*}{ Subtype } & $n$ & \multicolumn{2}{c}{ Gender } & \multicolumn{5}{c}{ Age (years) } \\
& & Male & Female & Mean (Stderr) & $>40$ & $20-40$ & $\leq 20$ \\
\hline 1a & 4 & 4 & & $31(3.37)$ & 4 & 4 \\
1b & 31 & 15 & 16 & $41.3(11.25)$ & 17 & 13 & 1 \\
2a & 8 & 3 & 5 & $45.5(17.44)$ & 4 & 4 & \\
3a & 6 & 4 & 2 & $39.7(13.49)$ & 3 & 3 & \\
3b & 16 & 10 & 6 & $35.1(9.66)$ & 3 & 13 & \\
6a & 35 & 28 & 7 & $33.6(8.44)$ & 6 & 27 & 2 \\
Total & 100 & 64 & 36 & $37.5(11.22)$ & 33 & 64 & 3 \\
\hline
\end{tabular}


distribution has been described in two studies, one by us [31, 32] and the other by Dunford et al. [33], both of which argue against the possibility of origin of $3 \mathrm{~b}$ in Vietnam. If, as we suggest, subtype $3 \mathrm{~b}$ originated in Yunnan in the southwest, the strains must have been brought to Hainan Island via the IDU network that runs through Guangxi province. In a previous report, a strong association between $3 \mathrm{~b}$ strains and IDUs in Guangxi province was indicated [28].

Compared to that described previously in Haikou City on Hainan Island, the frequency of subtype $1 \mathrm{~b}$ isolates in this study decreased from $62.5 \%$ to $30.6 \%$ and that of subtype $2 \mathrm{a}$ from $29.2 \%$ to $9.9 \%$. During 1992-2002, there was a significant linear decrease, by $2.3 \%$ annually, in the frequency of $1 \mathrm{~b}$ strains detected in Belgium [34]. A similar decrease in subtype $1 \mathrm{~b}$ isolates was also seen in Asian countries and in Australia [35]. It has been shown in Europe that $1 \mathrm{~b}$ and $2 \mathrm{a}$ isolates are more frequently detected in older patients with a history of blood transfusion [36, 37]. Such an age association was also reported in one of our recent studies on patients from Guangdong province [16]. It was described in another study that the most common risk factors of HCV infection with genotype 1 or 2 strains were unsafe medical procedures such as blood transfusion ( $67 \%$ and $62 \%$ of patients, respectively), surgery $(19.9 \%$ and $20.4 \%$ ), and dental treatment (14.6\% and $17.5 \%$ ) [11]. However, these risks are now becoming relatively low in China as sterilization measures are improved for these medical procedures [38]. Therefore, we strongly believe that the observed decrease in subtypes $1 \mathrm{~b}$ and $2 \mathrm{a}$ in this study should be seen in the context of a substantial relative increase in other HCV lineages, such as $3 \mathrm{~b}$ and $6 \mathrm{a}$, as described above. The latter two subtypes may have undergone more efficient dissemination in recent years mainly due to the rapidly growing number of IDUs in China. Nevertheless, the improvements in the safety of blood transfusion and the implementation of a voluntary blood donation system in China since 1996 should be reflected in our results [38].

In this study, we detected 1 a strains in $4 \%$ of patients sampled. This frequency is higher than that we have recently reported on patients from various regions of China [13-19] , and this may represent a sampling variation. If not, it suggests a recent, increasing trend of $1 \mathrm{a}$ prevalence in the general population on Hainan Island. It is well known that $1 \mathrm{a}$ is the major genetic lineage of HCV in the US [39]. In contrast, it is rare in China [14] and in some other Asian countries [40], although it has been frequently detected in Thailand [41] and even more so in the Philippines [42]. In the latter two countries, the majority $1 \mathrm{a}$ strains might have their origins in the US [31]. Unlike the 3b strains described above, Guangdong and Guangxi provinces could be the source regions of $1 \mathrm{a}$ isolates detected on Hainan Island, as suggested by the tree shown in Fig. 3. However, the main transmission route responsible for their dissemination has yet to be discovered. Although no history of IDU was declared by any of the patients carrying these 1a strains, the phylogeographic tree indicated a strong similarity to those 1a strains characterized among IDUs from Guangxi province [28]. Analysis of the 1a sequences hitherto obtained in China showed that the majority segregated into cluster A, while within cluster A most were from IDUs in Guangxi province. These lines of evidence suggest a close association of 1a with IDU in China. Apparently, cluster A represents the 1a strains that began to circulate in Guangxi province and then spread to neighboring Guangdong and Hainan, with a few distributed to other regions. Such a wide range of dissemination must have been assisted by IDU network transmission, although we have no evidence to confirm this. Compared to cluster A, cluster C includes 1a strains that are almost entirely associated with IDUs, representing a more robust example of this mode of transmission. Overall, a variety of geographic origins are indicated, but only a single 1a strain was sampled in the northern part of China. This might be due to under-sampling of patients and the absence of a wide-ranging survey of HCV among IDUs in the regions. Given our results on migration trends, a more interesting question is raised: when was subtype 1a most likely introduced into China? By scaling branch length, we estimated a set of time points at which different $1 \mathrm{a}$ branches diverged in the tree. A time frame of 1999-2004 was indicated during which these 1a strains were probably transmitted into new hosts. Surprisingly, this time frame was much later than that we estimated for other HCV subtypes. Although we should bear in mind that

\section{KARGER}




\section{Cellular Physiology Cell Physiol Biochem 2016;39:316-330 \begin{tabular}{l|l} 
DOI: 10.1159/000445626 & $\begin{array}{l}\text { O 2016 The Author(s). Published by S. Karger AG, Basel } \\
\text { www.karger.com/cpb }\end{array}$ \\
\cline { 2 - 4 }
\end{tabular} \\ Wu et al.: HCV Distribution Pattern on Hainan Island}

the time frames of other HCV subtypes correspond to a period during which a procedural error occurred in an officially encouraged plasma campaign in China [17], a delayed time frame for subtype 1a may indicate its unique epidemic history. A better interpretation was provided by a BSP, which estimated the rapid population growth of subtype 1a occurred during 1998 2005. Although followed by a gradual slowing, this growth continues to the present day. This pattern is remarkably different from those we estimated for $1 \mathrm{~b}, 2 \mathrm{a}$, $3 \mathrm{a}, 3 \mathrm{~b}$, and $6 \mathrm{a}$ [17] and may indicate a different history of the virus dissemination that is determined by the major transmission route, which we have suggested is through the IDU network. Further, the continued growth of 1 a implies that 1 a dissemination in China remains uncontrolled, although efforts have been made to improve the safety of blood transfusion in the country since 1996 [38].

\section{Acknowledgments}

Ling Lu was supported by a grant from the National Institute of Allergy and Infectious Diseases (5 R01 AI080734). Tao Wu was supported by a grant from Hainan Provincial government (ZDXM2015072). Yuanping Zhou was supported by a grant from the National Natural Sciences Foundation of China (81470856). The funding agencies had no role in study design, data collection and analysis, decision to publish, or preparation of the manuscript.

\section{Disclosure Statement}

The authors declare that there are no conflicts of interest.

\section{References}

1 No authors listed: Hepatitis C. Wkly Epidemiol Rec 1997;72:65-69.

2 Smith DB, Bukh J, Kuiken C, Muerhoff AS, Rice CM, Stapleton JT, Simmonds P: Expanded classification of hepatitis $C$ virus into 7 genotypes and 67 subtypes: Updated criteria and genotype assignment web resource. Hepatology 2014;59:318-327.

3 Simmonds P, Bukh J, Combet C, Deleage G, Enomoto N, Feinstone S, Halfon P, Inchauspe G, Kuiken C, Maertens G, Mizokami M, Murphy DG, Okamoto H, Pawlotsky JM, Penin F, Sablon E, Shin IT, Stuyver LJ, Thiel HJ, Viazov S, Weiner AJ, Widell A: Consensus proposals for a unified system of nomenclature of hepatitis C virus genotypes. Hepatology 2005;42:962-973.

4 Feld JJ, Hoofnagle JH: Mechanism of action of interferon and ribavirin in treatment of hepatitis C. Nature 2005;436:967-972.

5 Manns MP, Wedemeyer H, Cornberg M: Treating viral hepatitis C: efficacy, side effects, and complications. Gut 2006;55:1350-1359.

6 Delang L, Neyts J, Vliegen I, Abrignani S, Neddermann P, De Francesco R: Hepatitis C virus-specific directly acting antiviral drugs. Curr Top Microbiol Immunol 2013;369:289-320.

7 Gower E, Estes C, Blach S, Razavi-Shearer K, Razavi H: Global epidemiology and genotype distribution of the hepatitis C virus infection. J Hepatol 2014;61:45-57.

8 Wieland, SF: The chimpanzee model for hepatitis B virus infection. Cold Spring Harb Perspect Med DOI:10.1101/cshperspect.a021469.

9 Li C, Barnes E, Newton PN, Fu Y, Vongsouvath M, Klenerman P, Okamoto H, Abe K, Pybus OG, Lu L: An expanded taxonomy of hepatitis $\mathrm{C}$ virus genotype 6: Characterization of 22 new full-length viral genomes. Virology 2015;476:355-363. 


\section{Cellular Physiology Cell Physiol Biochem 2016;39:316-330 \begin{tabular}{l|l} 
DOI: 10.1159/000445626 & and Biochemistry \\
Published online: June 29, 2016 & $\begin{array}{l}\text { 2016 The Author(s). Published by S. Karger AG, Basel } \\
\text { www.karger.com/cpb }\end{array}$
\end{tabular} \\ Wu et al.: HCV Distribution Pattern on Hainan Island}

10 Pybus OG, Rambaut A: Evolutionary analysis of the dynamics of viral infectious disease. Nat Rev Genet 2009;10:540-550.

11 Rao H, Wei L, Lopez-Talavera JC, Shang J, Chen H, Li J, Xie Q, Gao Z, Wang L, Wei J, Jiang J, Sun Y, Yang R, Li H, Zhang H, Gong Z, Zhang L, Zhao L, Dou X, Niu J, You H, Chen Z, Ning Q, Gong G, Wu S, Ji W, Mao Q, Tang H, Li S, Wei S, Sun J, Lu L, Jia J, Zhuang H: Distribution and clinical correlates of viral and host genotypes in Chinese patients with chronic hepatitis C virus infection. J Gastroenterol Hepatol 2014;29:545-553.

12 An Y, Wu T, Wang M, Lu L, Li C, Zhou Y, Fu Y, Chen G: Conservation in China of a novel group of HCV variants dating to six centuries ago. Virology 2014;464-465:21-25.

13 Lu L, Wang M, Xia W, Tian L, Xu R, Li C, Wang J, Rong X, Xiong H, Huang K, Huang J, Nakano T, Bennett P, Zhang Y, Zhang L, Fu Y: Migration patterns of hepatitis C virus in china characterized for five major subtypes based on samples from 411 volunteer blood donors from 17 provinces and municipalities. J Virol 2014;88:7120-7129.

14 Fu Y, Wang Y, Xia W, Pybus OG, Qin W, Lu L, Nelson K: New trends of HCV infection in China revealed by genetic analysis of viral sequences determined from first-time volunteer blood donors. J Viral Hepat 2011;18:42-52.

15 Fu Y, Qin W, Cao H, Xu R, Tan Y, Lu T, Wang H, Tong W, Rong X, Li G, Yuan M, Li C, Abe K, Lu L, Chen G: HCV 6a prevalence in Guangdong province had the origin from Vietnam and recent dissemination to other regions of China: phylogeographic analyses. PLoS One 2012;7:e28006.

16 Gu L, Tong W, Yuan M, Lu T, Li C, Lu L: An increased diversity of HCV isolates were characterized among 393 patients with liver disease in China representing six genotypes, 12 subtypes, and two novel genotype 6 variants. J Clin Virol 2013;57:311-317.

17 Lu L, Tong W, Gu L, Li C, Lu T, Tee KK, Chen G: The current hepatitis C virus prevalence in China may have resulted mainly from an officially encouraged plasma campaign in the 1990s: a coalescence inference with genetic sequences. J Virol 2013;87:12041-12050.

18 Lu L, Nakano T, He Y, Fu Y, Hagedorn CH, Robertson BH: Hepatitis C virus genotype distribution in China: predominance of closely related subtype $1 \mathrm{~b}$ isolates and existence of new genotype 6 variants. J Med Virol 2005;75:538-549.

19 Xia X, Lu L, Tee KK, Zhao W, Wu J, Yu J, Li X, Lin Y, Mukhtar MM, Hagedorn CH, Takebe Y: The unique HCV genotype distribution and the discovery of a novel subtype $6 \mathrm{u}$ among IDUs co-infected with HIV-1 in Yunnan, China. J Med Virol 2008;80:1142-1152.

20 Posada, D: Selection of models of DNA evolution with jModelTest. Methods Mol Biol 2009;537:93-112.

21 Tamura K, Stecher G, Peterson D, Filipski A, Kumar S: MEGA6: Molecular Evolutionary Genetics Analysis version 6.0. Mol Biol Evol 2013;30:2725-2729.

22 Drummond AJ, Rambaut A: BEAST: Bayesian evolutionary analysis by sampling trees. BMC Evol Biol 2007;7:214.

23 Pybus OG, Barnes E, Taggart R, Lemey P, Markov PV, Rasachak B, Syhavong B, Phetsouvanah R, Sheridan I, Humphreys IS, Lu L, Newton PN, Klenerman P: Genetic history of hepatitis C virus in East Asia. J Virol 2009;83:1071-1082.

24 Markov PV, Pepin J, Frost E, Deslandes S, Labbe AC, Pybus OG: Phylogeography and molecular epidemiology of hepatitis C virus genotype 2 in Africa. J Gen Virol 2009;90:2086-2096.

25 Yuan M, Lu T, Li C, Lu L: The evolutionary rates of HCV estimated with subtype 1a and 1b sequences over the ORF length and in different genomic regions. PLoS One 2013;8:e64698.

26 Du J, Wang Z, Xie B, Zhao M: Hepatitis C knowledge and alcohol consumption among patients receiving methadone maintenance treatment in Shanghai, China. Am J Drug Alcohol Abuse 2012;38:228-232.

27 Peng JS, Wang X, Liu MQ, Zhou DJ, Gong J, Xu HM, Chen JP, Zhu HH, Zhou W, Ho WZ: Genetic variation of hepatitis C virus in a cohort of injection heroin users in Wuhan, China. Virus Res 2008;135:191-196.

28 Garten RJ, Zhang J, Lai S, Liu W, Chen J, Yu XF: Coinfection with HIV and hepatitis C virus among injection drug users in southern China. Clin Infect Dis 2005;41:S18-24.

29 Xu R, Tong W, Gu L, Li C, Fu Y, Lu L: A panel of 16 full-length HCV genomes was characterized in China belonging to genotypes 1-6 including subtype $2 \mathrm{f}$ and two novel genotype 6 variants. Infect Genet Evol 2013;20:225-229.

30 Sheng L, Cao WK: HIV/AIDS epidemiology and prevention in China. Chin Med J (Engl) 2008;121:12301236. 


\section{Cellular Physiology Cell Physiol Biochem 2016;39:316-330 \begin{tabular}{ll|l} 
DOI: 10.1159/000445626 & $\begin{array}{l}\text { O 2016 The Author(s). Published by S. Karger AG, Basel } \\
\text { www.karger.com/cpb }\end{array}$
\end{tabular} \\ Wu et al.: HCV Distribution Pattern on Hainan Island}

31 Li C, Yuan M, Lu L, Lu T, Xia W, Pham VH, Vo AX, Nguyen MH, Abe K: The genetic diversity and evolutionary history of hepatitis C virus in Vietnam. Virology 2014;468:197-206.

32 Pham VH, Nguyen HD, Ho PT, Banh DV, Pham HL, Pham PH, Lu L, Abe K: Very high prevalence of hepatitis C virus genotype 6 variants in southern Vietnam: large-scale survey based on sequence determination. Jpn J Infect Dis 2011;64:537-539.

33 Dunford L, Carr MJ, Dean J, Waters A, Nguyen LT, Ta Thi TH, Thi LA, Do HD, Thi TT, Nguyen HT, Diem Do TT, Luu QP, Connell J, Coughlan S, Hall WW, Nguyen Thi LA: Hepatitis C virus in Vietnam: high prevalence of infection in dialysis and multi-transfused patients involving diverse and novel virus variants. PLoS One 2012; 7:e41266.

34 Gerard C, Delwaide J, Vaira D, Bastens B, Servais B, Wain E, Bataille C, Daenen G, Belaiche J: Evolution over a 10 year period of the epidemiological profile of 1,726 newly diagnosed HCV patients in Belgium. J Med Virol 2005;76:503-510.

35 Maieron A, Metz-Gercek S, Hackl F, Luger C, Ziachehabi A, Strauss R, Schofl R, Mittermayer H: Chronic hepatitis C in Austria, 1992-2006: genotype distribution and demographic factors. Euro Surveill 2010;15:19492.

36 Silini E, Bono F, Cividini A, Cerino A, Maccabruni A, Tinelli C, Bruno S, Bellobuono A, Mondelli M: Molecular epidemiology of hepatitis C virus infection among intravenous drug users. J Hepatol 1995; 22: 691-695.

37 Dubois F, Desenclos JC, Mariotte N, Goudeau A: Hepatitis C in a French population-based survey, 1994: seroprevalence, frequency of viremia, genotype distribution, and risk factors. The Collaborative Study Group. Hepatology 1997;25:1490-1496.

38 Shan H, Wang JX, Ren FR, Zhang YZ, Zhao HY, Gao GJ, Ji Y, Ness PM: Blood banking in China. Lancet 2002;360:1770-1775.

39 Nainan OV, Alter MJ, Kruszon-Moran D, Gao FX, Xia G, McQuillan G, Margolis HS: Hepatitis C virus genotypes and viral concentrations in participants of a general population survey in the United States. Gastroenterology 2006;131:478-484.

40 Lwin AA, Shinji T, Khin M, Win N, Obika M, Okada S, Koide N: Hepatitis C virus genotype distribution in Myanmar: Predominance of genotype 6 and existence of new genotype 6 subtype. Hepatol Res 2007;37:337-345.

41 Jutavijittum P, Jiviriyawat Y, Yousukh A, Pantip C, Maneekarn N, Toriyama K: Genotypic distribution of hepatitis $\mathrm{C}$ virus in voluntary blood donors of northern Thailand. Southeast Asian J Trop Med Public Health 2009;40:471-479.

42 Katayama Y, Barzaga NG, Alipio A, Soetjipto, Doi H, Ishido S, Hotta H: Genotype analysis of hepatitis C virus among blood donors and inmates in Metro Manila, The Philippines. Microbiol Immunol 1996;40:525-529. 